DOI: $10.17516 / 1999-494 X-0224$

УДК 519.816

\title{
A Mathematical Model of the Detection Probability of the Point Target by the Operator of the Payload Electro-Optical System for Unmanned Aerial Vehicle
}

\author{
Aleksey I. Tishchenko and Stepan V. Artyshchenko* \\ Military Education and Research Centre of Military-Air Forces \\ "Military-Air Academy \\ Named After Professor N.E. Zhukovsky and Yu.A. Gagarin» \\ Voronezh, Russian Federation
}

Received 27.09.2019, received in revised form 04.12.2019, accepted 21.01.2020

Abstract. The paper deals with a mathematical model of the probability of detection of a point target by the operator of the payload of an optoelectronic device installed on an unmanned aerial vehicle, based on the use of the Johnson criterion and the binomial law of repeated tests.

Keywords: a mathematical model, a probability of detection, optoelectronic systems, unmanned aerial systems, a payload operator, rescue operations.

Citation: Tishchenko A.I., Artyshchenko S.V. A mathematical model of the detection probability of the point target by the operator of the payload electro-optical system for unmanned aerial vehicle, J. Sib. Fed. Univ. Eng. \& Technol., 2020, 13(3), 328-337. DOI: 10.17516/1999-494X-0224

(C) Siberian Federal University. All rights reserved

This work is licensed under a Creative Commons Attribution-Non Commercial 4.0 International License (CC BY-NC 4.0).

* Corresponding author E-mail address: aleksei.tishenko@yandex.ru 


\title{
Математическая модель вероятности обнаружения \\ точечной цели оператором полезной нагрузки \\ оптико-электронной системы \\ беспилотного летательного аппарата
}

\author{
А.И. Тищенко, С.В. Артыщенко \\ Военный учебно-научный изентр Военно-воздушных сил \\ «Военно-воздушная академия \\ имени профессора Н.Е. Жуковского и Ю.А. Гагарина» \\ Российская Федераџия, Воронеж
}

Аннотация. В работе рассматривается математическая модель вероятности обнаружения точечной цели оператором полезной нагрузки оптико-электронного средства, установленного на беспилотном летательном аппарате, на основе использования критерия Джонсона и биноминального закона повторных испытаний.

Ключевые слова: математическая модель, вероятность обнаружения, оптико-электронные системы, беспилотные летательные системы, оператор полезной нагрузки, аварийноспасательные работы.

Цитирование: Тищенко, А.И. Математическая модель вероятности обнаружения точечной цели оператором полезной нагрузки оптико-электронной системы беспилотного летательного аппарата / А.И. Тищенко, С.В. Артыщенко // Журн. Сиб. федер. ун-та. Техника и технологии, 2020. 13(3). С. 328-337. DOI: 10.17516/1999494X-0224

\section{Введение}

Подразделения поисково-спасательной службы используют беспилотные летательные аппараты (БпЛА) при проведении аварийно-спасательных работ в тех случаях, когда необходимо оперативно, достоверно без риска для жизнедеятельности личного состава спасательного подразделения, экипажей самолетов и вертолетов оценить ущерб от чрезвычайных ситуаций природного или техногенного характера, а также при поиске и спасении пропавших или терпящих бедствие людей в труднодоступных районах.

В зависимости от решаемой задачи на БпЛА устанавливается соответствующая целевая нагрузка. Для поиска людей используют видео и инфракрасные (ИК) камеры, которые в комплексе с оборудованием беспилотного летательного аппарата можно рассматривать как оптико-электронные системы (ОЭС).

Для планирования мероприятий, связанных с применением БпЛА в ходе выполнения аварийно-спасательных работ, штабу поисково-спасательных работ целесообразно иметь расчетные программы, позволяющие с высокой степенью достоверности, исходя из наличия и возможностей ОЭС, парка беспилотных летательных аппаратов прогнозировать сроки выполнения поставленной задачи.

Реализация расчетных программ возможна при наличии математических моделей, соответствующих задачам, стоящим перед расчетом БпЛА в ходе проведения аварийно-спасательных работ. 
Целью данной работы является разработка математической модели расчета вероятностей обнаружения и распознавания наземной цели оператором полезной нагрузки оптико-электронной системы, установленной на БпЛА, осуществляющем в заданном районе случайный поиск. Обнаружение и распознавание оператором осуществляется по монитору наземного пункта управления БпЛА.

Для решения поставленной цели определимся с некоторыми понятиями, которые будем использовать в дальнейшем.

Под наземным объектом поиска будем понимать простые, малоразмерные, стационарные или малоподвижные объекты, находящиеся на поверхности Земли. Площадь этих объектов, как правило, в сотни тысяч раз меньше площади районов поиска. Поэтому в дальнейшем объекты поиска будем называть точечными.

Для подразделения поисково-спасательных работ, осуществляющего поиск людей (объектов поиска) с помощью ОЭС БпЛА, основными визуальными задачами являются обнаружение и распознавание объекта поиска. Под обнаружением будем понимать уровень восприятия, при котором осуществляется выделение на экране монитора пятна, соответствующего объекту поиска, на фоне помех. Под распознаванием будем понимать уровень восприятия, при котором объект поиска различается уровнем детализации, позволяющим определить его класс - человек, автомобиль и т.д.

Тактическими характеристиками задачи являются высота, маршрут полета БпЛА в районе поиска, а также время обнаружения и распознавания объекта поиска.

На решение задачи по обнаружению и распознаванию объектов поиска могут оказать влияние следующие факторы: качество изображения объекта поиска на экране ОЭС; объем априорной информации, на основе которой осуществляется выделение объекта поиска на фоне окружающей среды; степень подготовки оператора полезной нагрузки. Первые три фактора определяются параметрами ОЭС и БпЛА, привлекаемых к поиску. К числу технических характеристик ОЭС, оказывающих влияние на вероятности обнаружения и распознавания наземных объектов, на выбор маршрута и профиль полета БпЛА, относятся: фокусное расстояние, углы поля зрения по горизонтали и вертикали и разрешающая способность объектива; размер пикселя и матрицы приемника излучения.

\section{Математическая модель вероятности обнаружения цели оператором полезной нагрузки оптико-электронной системы беспилотного летательного аппарата}

Решение поставленной цели выполним применительно к следующим условиям. Расчет беспилотного летательного аппарата осуществляет поиск точечного наземного объекта площадью $S_{m}$ в заданном районе поиска площадью $S_{p}$ с помощью ОЭС видимого диапазона, установленной на БпЛА. ОЭС включает в себя видеокамеру и монитор. Монитор находится на наземном пункте управления беспилотного летательного аппарата. Формат изображения монитора полностью соответствует формату изображения приемника излучения. Видеокамера установлена непосредственно на летательном аппарате под углом визирования $\alpha$. Технические характеристики камеры с установленным объективом: вертикальный угол поля зрения $\alpha_{s}$; горизонтальный угол поля зрения $\alpha_{2}$; фокусное расстояние объектива $f$; скорость съемки $\tau$ (для 
фотосъемки серийность съемки, для видеосъемки частота кадра); линейное разрешение ОЭС на местности $R$; размер пикселя матрицы $\delta$; размер матрицы приемника излучения по горизонтали $a$ и по вертикали $b$.

Проекция поля зрения видеокамеры на контролируемую в процессе полета БпЛА земную поверхность при угле визирования $0^{\circ}$ имеет форму прямоугольника, а при угле визирования, отличном от $0^{\circ}$, - трапецию (рис. 1) с параметрами и площадью, определяемыми следующими выражениями:

$$
\begin{aligned}
& l_{1}=A B=\frac{2 h_{\text {no: }} \operatorname{tg}\left(\frac{\alpha_{2}}{2}\right)}{\cos \left(\alpha+\frac{\alpha_{b}}{2}\right)}, \alpha+\frac{\alpha_{6}}{2}<90^{\circ} \\
& l_{2}=D C=\frac{2 h_{\text {non }} \operatorname{tg}\left(\frac{\alpha_{2}}{2}\right)}{\cos \left(\alpha-\frac{\alpha_{6}}{2}\right)}, \alpha-\frac{\alpha_{b}}{2}<-90^{\circ}
\end{aligned}
$$

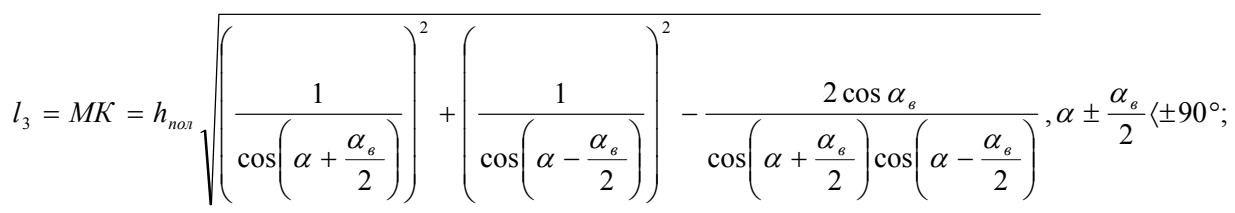

$$
\begin{aligned}
& l_{4}=P G=\frac{l_{2}-l_{1}}{2} ; \\
& S_{\text {обз }}=l_{4} l_{3},
\end{aligned}
$$

где $l_{1}$ - основание трапеции (сторона прямоугольника), ближнее относительно БпЛА; $l_{2}$ - основание трапеции (сторона прямоугольника), дальнее относительно БпЛА; $l_{3}$ - высота трапеции (прямоугольника); $l_{4}$ - средняя линяя трапеции; $S_{\text {обз }}$ площадь зоны обзора ОЭС земной поверхности; $h_{\text {пол }}$ - высота полета беспилотного летательного аппарата.

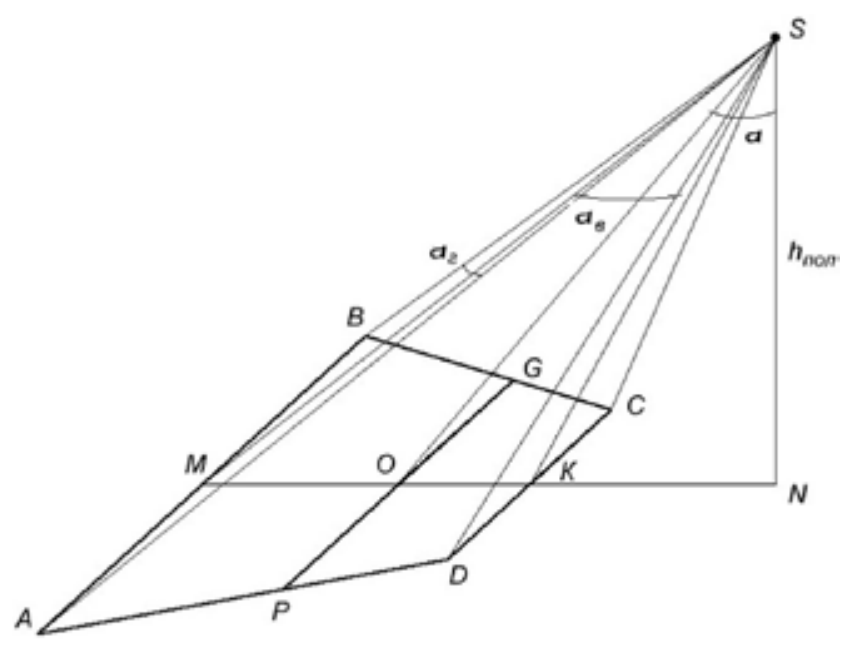

Рис. 1. Проекция поля зрения видеокамеры на земную поверхность

Fig. 1. Projection of the field of view of the camera on the earth's surface 
Связь между размерами матрицы приемника излучения и углами поля зрения ОЭС такова:

$$
\begin{aligned}
& \alpha_{2}=\operatorname{arctg} \frac{a}{2 f} \\
& \alpha_{B}=\operatorname{arctg} \frac{b}{2 f} .
\end{aligned}
$$

Решение задачи рассмотрим применительно к случаю, когда количество БпЛА, привлекаемых к поиску, ограничено, район поиска обширен, данные о месте и маршруте движения терпящих бедствие отсутствует. В подобных условиях для детального осмотра участков местности в пределах района поиска используют прямолинейные взаимно параллельные маршруты - параллельное галсирование. Маршруты прокладывают таким образом, чтобы края поля зрения ОЭС БпЛА двух соседних маршрутов при установленной высоте полета

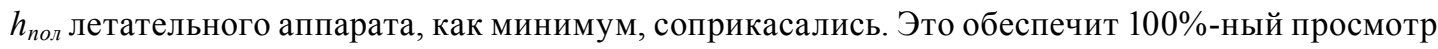
ОЭС БпЛА района поиска. Следовательно, расстояние между маршрутами должно быть равным $l_{l}$. Облет района поиска летательным аппаратом осуществляется с крейсерской скоростью $V_{\kappa p}$.

В процессе полета БпЛА получаемое с помощью видеокамеры видеоизображение участка местности передается по радиоканалу на наземный пункт управления, где отображается на мониторе оператора целевой нагрузки. Оператор в ходе анализа полученной информации обнаруживает цель.

В системах видеорегистрации для определения вероятностей обнаружения и распознавания объектов используют критерий Джонсона. Его суть применительно к решаемой задаче заключается в том, что существует соответствие между числом разрешаемых периодов эквивалентной миры, укладывающихся на минимальном размере наблюдаемого объекта поиска, и уровнем его восприятия. В качестве эквивалентной миры взята штриховая мира прямоугольной формы, ширина которой равна минимальному размеру объекта поиска, а длина соответствует размеру, перпендикулярному минимальному размеру. Этот критерий также применяется для оптико-электронных систем с выходным видеосигналом видеокамер с тем отличием, что вместо пар линий штриховой миры значения указывают в телевизионных линиях или, что то же самое, в элементах изображения (пикселях). Графическое выражение критерия Джонсона применительно к решаемой задаче обнаружения и распознавания изображений объектов поиска представлено на рис. 2 [1].

Используя метод наименьших квадратов и аппроксимируя кривые вероятностей обнаружения и распознавания объектов от количества элементов разрешения занимаемыми ими на видеоматериалах экспоненциальными функциями, получим аналитические зависимости:

$$
\begin{aligned}
& P_{o \tilde{\sigma}}=1-e^{-0,2(N-1)^{2}}, \\
& P_{p a c}=1-e^{-0,015(N-1)^{2}},
\end{aligned}
$$

где $P_{\text {об, }} P_{p a c}$ - вероятности обнаружения и распознавания и идентификации объектов; $N-$ количество элементов разрешения занимаемых объектом поиска на мониторе наземного пункта управления. 


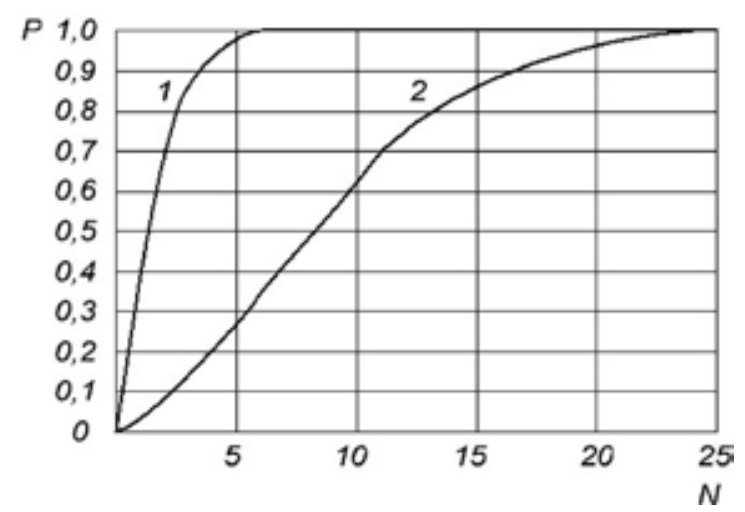

Рис. 2. Зависимости вероятностей обнаружения (1) и распознавания (2) объектов поиска от количества элементов разрешения, занимаемых ими на мониторе

Fig. 2. Dependences of probabilities of detection (1) and recognition (2) of search objects the number of resolution elements they occupy on the monitor

Зависимость между размерами объекта поиска на местности и количеством $N$ определяется выражением [2]

$$
N=\frac{l_{m} f}{R h_{\text {пол }}}
$$

где $l_{m}$ - линейные размеры объекта поиска по направлению полета БпЛА; $R$ - линейное разрешение ОЭС на местности, связанное с размерами $\delta$ соотношением вида

$$
R=\frac{h_{\text {пол } \delta}}{f} \text {. }
$$

При попадании объекта поиска в поле зрения ОЭС он появится на экране монитора наземного пункта управления и оператор полезной нагрузки обнаружит и распознает объект поиска с вероятность $p$, равной вероятности распознавания $P_{p a c}$.

Район поиска применительно к ОЭС БПЛА можно рассматривать как серию видеокадров $n=n_{1} n_{2}-$ независимых испытаний, определяемых произведением количества элементов разрешения $n_{l}$, приходящимся на полный маршрут полета БпЛА, и количеством кадров $n_{2}$, приходящихся на элемент разрешения, определяемых выражениями (12), (13). Элемент разрешения представляет собой поле зрения ОЭС на земную поверхность.

$$
\begin{aligned}
& n=n_{1} n_{2}, \\
& n_{1}=\left\lceil\frac{l_{\text {nол }}}{l_{3}}\right\rceil, \\
& n_{2}=\left\lceil\frac{l_{3}}{V_{\kappa p}}\right\rceil \tau,
\end{aligned}
$$

где $l_{\text {пол }}$ - полная протяженность полета БпЛА в районе поиска; $\lceil x\rceil$ - наибольшее целое, большее или равное $x$.

Количество кадров $m$, на которых имеется изображение объекта поиска, определяется произведением количества элементов разрешения $n_{3}$, приходящимся на размер объекта поиска, и 
количеством кадров $n_{2}$, приходящихся на элемент разрешения, определяемых выражениями (13), (14).

$$
\begin{aligned}
& m=n_{3} n_{2}, \\
& n_{3}=\left\lceil\frac{l_{m}}{l_{3}}\right\rceil .
\end{aligned}
$$

Если обозначить вероятность обнаружения и распознавания объекта поиска в $i$-м снимке через $p_{i}$, равное $p$, то для расчета вероятности того, что в $n$ снимках района поиска обнаружение и распознавание объекта поиска произойдут не менее чем в $m$ снимках можно использовать биноминальный закон повторных испытаний [2]:

$$
P_{n}(m)=C_{n}^{m} p^{m}(1-p)^{n-m}
$$

где $P_{n}(m)$ - вероятность обнаружения объекта поиска оператором полезной нагрузки $m$ раз в серии из $n$ видеокадров; $C_{n}^{m}$ - число сочетаний из $n$ по $m$, равное

$$
C_{n}^{m}=\frac{n !}{m !(n-m) !}
$$

Используя $P_{n}(m)$ можно вычислить вероятность обнаружения и распознавания объекта поиска по видеоматериалам хотя бы 1 раз:

$$
R_{1, n}=1-\sum_{j=0}^{0} P_{0, n}=1-\prod_{i=1}^{n} q_{j},
$$

где $q_{i}=1-p_{i}, p_{i}=0$ для $i=1-z ; p_{i}=P_{\text {расі }}$ для $i=z+1-z+m ; p_{i}=0$ для $i=z+m-n$.

Отсюда

$$
R_{1, n}=1-\left(P_{p a c z+1} P_{p a c z+2} \cdots P_{p a c z+m}\right)
$$

Так как $P_{p a c z+1}=P_{p a c z+2}=\ldots=P_{p a c z+m}$, то выражение (19) примет вид

$$
R_{1, n}=1-P_{p a c}^{m}
$$

Подставляя в (20) выражения (3), (6), (7), (9), (12) - (16), получим выражение, позволяющее оценить вероятность обнаружения и распознавания оператором полезной нагрузки цели с помощью установленных на БпЛА ОЭС в первом залете.

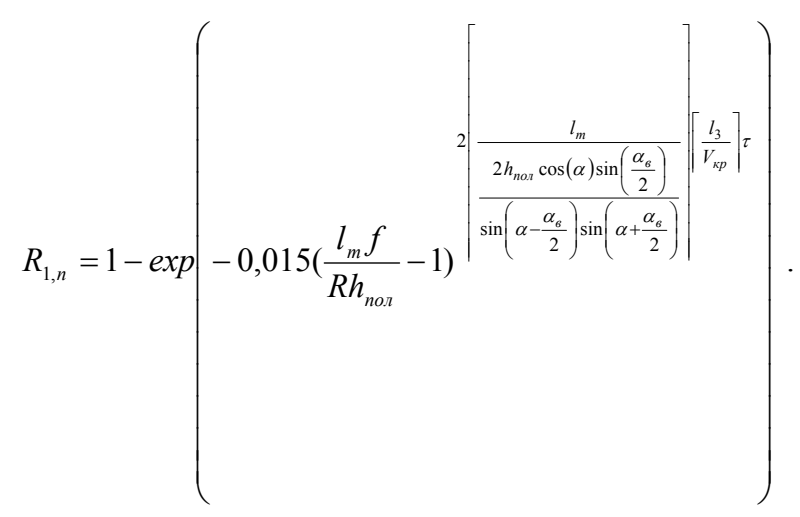


Время, необходимое для выполнения первого залета БпЛА в район поиска, определяется выражением

$$
t=\left(l_{\partial}+1+\pi \frac{l_{1}}{2}\right) \frac{l_{u}}{l_{1} V_{k p}}=\left(l_{z p}+1+\pi \frac{\frac{2 h_{\text {nол }} t g\left(\frac{\alpha_{2}}{2}\right)}{\sin \left(\alpha+\frac{\alpha_{b}}{2}\right)}}{2}\right) \frac{l_{u}}{\frac{2 h_{\text {nол }} t g\left(\frac{\alpha_{2}}{2}\right)}{\sin \left(\alpha+\frac{\alpha_{b}}{2}\right)} V_{k p}},
$$

где $l_{\partial}, l_{u}$ - размеры района поиска по длине и ширине соответственно.

Выражения (21) и (22) показывают, что вероятность обнаружения и распознавания объектов поиска в заданном районе и время, необходимое для выполнения этого поиска, можно рассчитать по известным характеристикам ОЭС, полета БпЛА, размерам района поиска и объекта поиска.

Эти зависимости позволяют решить обратную задачу, когда по заданному значению вероятности обнаружения и распознавания объекта поиска, известным характеристикам ОЭС, размерам района поиска и объекта поиска можно рассчитать параметры полета БпЛА и количество вылетов в район поиска, обеспечивающих минимальное время поиска.

С этой целью устанавливается пороговое значение $R_{l, n}{ }^{*}$. Из условия $R_{l, n}{ }^{*} \leq R_{l, n}$ применительно к размерам района и объекта поиска определяют характеристики ОЭС, полета БпЛА, при которых время поиска объекта будет минимальным.

Если в результате залета условие $R_{1, n}{ }^{*} \leq R_{l, n}$ не выполнимо, то в этом случае необходимо выполнить последующие залеты БпЛА в район поиска до тех пор, пока неравенство не будет выполнено.

Расчет $R_{l, k}$ в последующих залетах осуществляется с использованием выражения (23), а время $t$ определяется как произведение времени выполнения одного залета на количества залетов:

$$
R_{1, k}=1-\prod_{i=1}^{k}\left(1-R_{1, n}\right),
$$

где $k$ - количество залетов БпЛА в район поиска.

\section{Численное исследование математической модели}

Штат подразделения поисково-спасательных работ может содержать расчет БпЛА Phanton 3 Professional [3]. Поэтому численное исследование математической модели проведем применительно к данному беспилотному летательному аппарату, состоящему из непосредственно летательного аппарата, видеокамеры и пульта дистанционного управления.

Основные технические характеристики рассматриваемой модели:

беспилотный летательный аппарат: максимальная скорость полета 16 м/с ( 60 км/ч); максимальное время полета 23 мин; максимальная высота полета 6000 м; дальность передачи видеосигнала до 2000 м; подвес обеспечивает охват с углом наклона от -90 до 30; 
видеокамеры: оптика EXMOR 1/2,3"; эффективные пиксели 12,4 млн (всего пикселей 12,76 млн); объектив с углом обзора 94; фокусное расстояние 20 мм; эквивалентный формат 35 мм f/2,8; максимальный размер изображения 4000х3000; режим видеосъемки FHD: 1920x1020p 24/25/30/48/50/60 fps [4].

В качестве объекта поиска выберем человека, площадь поперечного сечения которого описывается овалом, длина и ширина которого составляют соответственно 0,75 и 0,5 м. Район поиска представим в виде квадрата со сторонами 2000 м, исходя из дальности передачи видеосигнала. Скорость полета БпЛА 60 км/ч. Пороговое значение вероятности обнаружения и распознавания объекта поиска по видеоматериалам хотя бы 1 раз определим равным 0,99 .

Результаты расчета времени, необходимого для обнаружения и распознавания расчетом БпЛА потерявшегося в районе поиска человека в зависимости от высоты полета летательного аппарата (50 - 1000 м), углов визирования видеокамеры 0, 10, 20, 30 градусов и технических характеристик, приведенных выше, представлены на рис. 3.

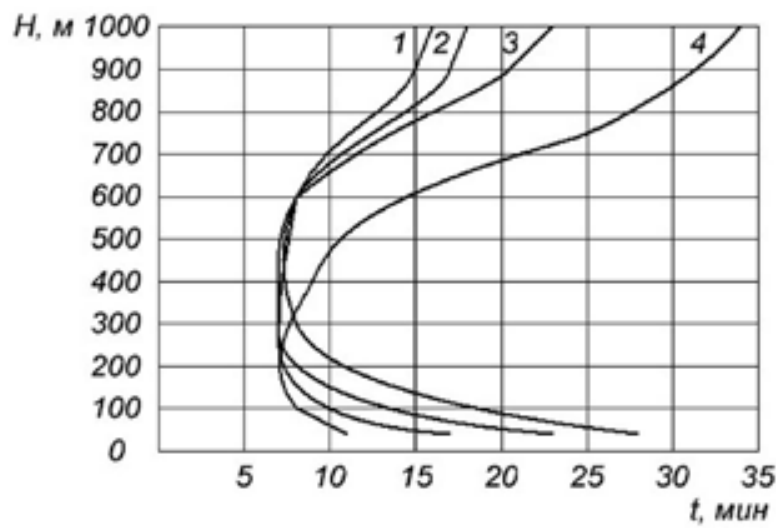

Рис. 3. Расчетные значения времени, необходимого для обнаружения и распознавания расчетом БпЛА потерявшегося в районе поиска человека при различных углах визирования $1-0^{\circ}, 2-10^{\circ}, 3-20^{\circ}, 4-30^{\circ}$

Fig. 3. Estimated time required for detection and calculation of the UAV lost in the search area of the person at different viewing angles $1-0^{\circ}, 2-10^{\circ}, 3-20^{\circ}, 4-30^{\circ}$

Анализ полученных результатов показывает, что время поиска потерявшихся людей в целом зависит от высоты полета летательного аппарата в ходе поиска и угла визирования видеокамеры. Наиболее существенна эта зависимость в диапазоне высот от 50 до 200 м и от 600 до 1000 м. В диапазоне высот от 200 до 600 м зависимость практически несущественно зависит от высоты полета летательного аппарата и угла визирования видеокамеры. Разность результатов не превышает 3 - 5 мин. Следовательно, можно сделать вывод, что поиск целесообразно вести на указанных высотах при угле визирования 0 градусов, так как при данном угле погрешности определения координат объекта по данным воздушной разведки минимальны. Полученные результаты согласуются с практическими рекомендациями, изложенными в [3], и указывают на адекватность представленной математической модели. 


\section{Заключение}

Таким образом, получена математическая модель вероятностей обнаружения и распознавания точечной цели оператором полезной нагрузки оптико-электронной системы беспилотного летательного аппарата. Она может быть реализована с применением программ высокого уровня. Результаты моделирования целесообразно использовать при планировании поисковоспасательных работ, связанных с поиском пропавших людей или объектов поиска в труднодоступных районах.

\section{Список литературы / References}

[1] Тищенко А.И., Ищук И.Н., Громов Ю.Ю. Определение высоты полета беспилотного летательного аппарата для выполнения видеонаблюдения с требуемой степенью детализации информации при поиске тепловых объектов, Информационно-сенсорные системы в теплофизических исследованиях: сб. науч. ст. (Тамбов, 6 - 9 ноября 2018 г.). Тамбов: Издательский центр ФГБОУ ВО «ТГТУ», 2018, 2, 190 - 194. [Tischenko A.I., Ischuk, I.N., Gromov Yu.Yu. Determination of the height of the flight of unmanned aircraft for video monitoring with the required degree of detail of information when searching for thermal objects, Information and sensor systems in thermophysical studies: collection of scientific works. article (Tambov, 6 - 9 November 2018). Tambov: Publishing house FGBOU VO «TSTU», 2018, 2, 190 - 194 (In Russian)]

[2] Корн Г., Корн Т. Справочник по математике для научных работников и инженеров, М.: Главная редакция физико-математической литературы, 1984. 832 с. [Korn G., Korn T. Handbook of mathematics for scientists and engineers, Moscow.: Home edition of physical and mathematical literature, 1984. 832 p. (In Russian)]

[3] Поисково-спасательные работы при помощи беспилотных летательных аппаратов [Электронный ресурс] - Режим доступа: https://fireman.club/statyi-polzovateley/poiskovospasatelnyih-rabotyi-pri-pomoshhi-bespilotnyih-letatelnyih-apparatov - Заглавие с экрана. [Search and rescue operations using unmanned aerial vehicles [Electronic resource] - Access: https://fireman. club/statyi-polzovateley/poiskovo-spasatelnyih-rabotyi-pri-pomoshhi-bespilotnyih-letatelnyihapparatov]

[4] Обзор квадрокоптера DJI Phanton 3 Professional. [Электронный pecypc] - Peжим доступа: https://mirquadrocopterov.ru/populyarnye-modeli/universalnye-drony/obzor-djiphantom-3-professional.html - Заглавие с экрана. [DJI Phantom 3 Professional. [Electronic resource] - Access: https://mirquadrocopterov.ru/populyarnye-modeli/universalnye-drony/obzor-djiphantom-3-professional.html] 